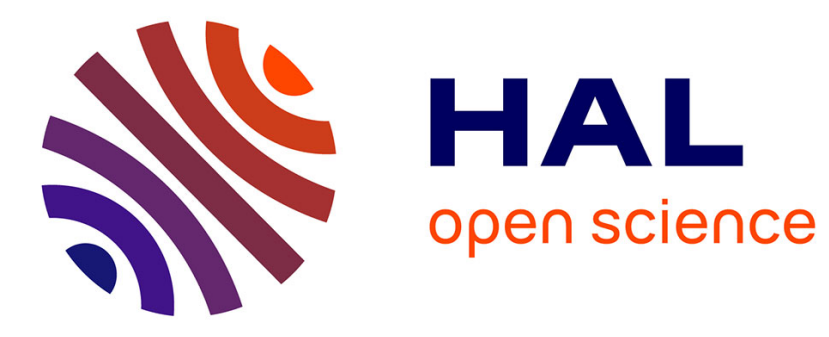

\title{
Self-similarity of strongly stratified inviscid flows
}

Paul Billant, Jean-Marc Chomaz

\section{To cite this version:}

Paul Billant, Jean-Marc Chomaz. Self-similarity of strongly stratified inviscid flows. Physics of Fluids, 2001, 13 (6), pp.1645-1651. 10.1063/1.1369125 . hal-01025349

HAL Id: hal-01025349

https://hal-polytechnique.archives-ouvertes.fr/hal-01025349

Submitted on 11 Sep 2014

HAL is a multi-disciplinary open access archive for the deposit and dissemination of scientific research documents, whether they are published or not. The documents may come from teaching and research institutions in France or abroad, or from public or private research centers.
L'archive ouverte pluridisciplinaire HAL, est destinée au dépôt et à la diffusion de documents scientifiques de niveau recherche, publiés ou non, émanant des établissements d'enseignement et de recherche français ou étrangers, des laboratoires publics ou privés. 


\section{AIP | Physics of Fluids}

\section{Self-similarity of strongly stratified inviscid flows}

Paul Billant and Jean-Marc Chomaz

Citation: Physics of Fluids (1994-present) 13, 1645 (2001); doi: 10.1063/1.1369125

View online: http://dx.doi.org/10.1063/1.1369125

View Table of Contents: http://scitation.aip.org/content/aip/journal/pof2/13/6?ver=pdfcov

Published by the AIP Publishing

\section{Articles you may be interested in}

Large-scale analysis of self-similar unstably stratified homogeneous turbulence

Phys. Fluids 26, 015110 (2014); 10.1063/1.4862445

Stratified shear flow instabilities at large Richardson numbers

Phys. Fluids 21, 054108 (2009); 10.1063/1.3147934

Dipolar eddies in a decaying stratified turbulent flow

Phys. Fluids 20, 026602 (2008); 10.1063/1.2842377

Self-similarity in decaying two-dimensional stably stratified adjustment

Phys. Fluids 19, 036603 (2007); 10.1063/1.2717514

Model dynamics and vertical collapse in decaying strongly stratified flows

Phys. Fluids 9, 2932 (1997); 10.1063/1.869405

\section{A|P| Journal of}

Journal of Applied Physics is pleased to announce André Anders as its new Editor-in-Chief 


\title{
Self-similarity of strongly stratified inviscid flows
}

\author{
Paul Billant ${ }^{\mathrm{a})}$ \\ LadHyX, CNRS, Ecole Polytechnique, F-91128 Palaiseau Cedex, France \\ and Météo-France CNRM Toulouse, 42 avenue Coriolis, F-31057 Toulouse, France \\ Jean-Marc Chomaz \\ LadHyX, CNRS, Ecole Polytechnique, F-91128 Palaiseau Cedex, France
}

(Received 8 May 2000; accepted 2 March 2001)

\begin{abstract}
It is well-known that strongly stratified flows are organized into a layered pancake structure in which motions are mostly horizontal but highly variable in the vertical direction. However, what determines the vertical scale of the motion remains an open question. In this paper, we propose a scaling law for this vertical scale $L_{v}$ when no vertical lengthscales are imposed by initial or boundary conditions and when the fluid is strongly stratified, i.e., when the horizontal Froude number is small: $F_{h}=U / N L_{h} \ll 1$, where $U$ is the magnitude of the horizontal velocity, $N$ the Brunt-Väisälä frequency and $L_{h}$ the horizontal lengthscale. Specifically, we show that the vertical scale of the motion is $L_{v}=U / N$ by demonstrating that the inviscid governing equations in the limit $F_{h} \rightarrow 0$, without any a priori assumption on the magnitude of $L_{v}$, are self-similar with respect to the variable $z N / U$, where $z$ is the vertical coordinate. This self-similarity fully accounts for the layer characteristics observed in recent studies reporting spontaneous layering from an initially vertically uniform flow. For such a fine vertical scale, vertical gradients are large, $O\left(1 / F_{h} L_{h}\right)$. Therefore, even if the magnitude of the vertical velocity is small and scales like $F_{h} U$, the leading order governing equations of these strongly stratified flows are not two-dimensional in contradiction with a previous conjecture. The self-similarity further suggests that the vertical spectrum of horizontal kinetic energy of pancake turbulence should be of the form $E\left(k_{z}\right) \propto N^{2} k_{z}^{-3}$, giving an alternative explanation for the observed vertical spectra in the atmosphere and oceans. (C) 2001 American Institute of Physics. [DOI: 10.1063/1.1369125]
\end{abstract}

\section{INTRODUCTION}

When a flow is strongly stably stratified the gravity acts as a restoring force and vertical displacements are inhibited. A ubiquitous feature of these flows observed in laboratory experiments $^{1-6}$ and numerical simulations ${ }^{7-10}$ is that the flow is predominantly horizontal but develops a strong vertical variability and reorganizes itself into decoupled horizontal layers. Such highly anisotropic layered turbulence has been most often called "pancake" turbulence but also "blini" or even "lasagne" turbulence by authors with different scientific tastes. Thin patches of turbulence and layers are also commonly observed in the oceans and atmosphere (see, for instance, Refs. 11 and 12).

Presently the vertical scaling law of the pancake turbulence remains an open issue. ${ }^{13}$ The well-known scaling analysis of Riley, Metcalfe, and Weissman ${ }^{7}$ and Lilly ${ }^{14}$ (hereafter, RMWL scaling analysis) for the nonlinear dynamics of pancake vortices does not predict any vertical scale for the motion since the flow becomes purely horizontal with undetermined vertical dependence in the strongly stratified limit. In the case where the pancake turbulence is observed after the gravitational collapse of three-dimensional homogeneous turbulence, ${ }^{8,15-17}$ the vertical scale of the motion, i.e.,

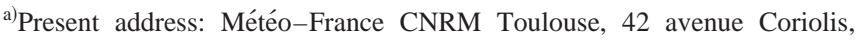
F-31057 Toulouse, France. Electronic mail: paul.billant@meteo.fr the layer thickness, could be a memory of the initial typical integral lengthscale of the turbulence existing before the collapse. ${ }^{18}$ In turbulent flows produced by a towed or an oscillatory grid, it can therefore depend on the mesh size. ${ }^{18}$ However, the numerical simulations by Herring and Métais ${ }^{9}$ and the vertical flat-plates wake experiments by Fincham, Maxworthy and Spedding ${ }^{4}$ have demonstrated that pancake structures can develop spontaneously from a basic flow which is initially uniform in the vertical direction. In these cases, the layer thickness scaling law cannot be related to the vertical geometrical constraints or to the initial integral lengthscale of the three-dimensional turbulence existing prior to the collapse. Rather, it should be an intrinsic feature solely determined by the externally imposed parameters, i.e., the characteristic horizontal velocity $U$, the horizontal lengthscale $L_{h}$ and the stratification through the BruntVäisälä frequency $N$. Diffusive effects are believed to be of minor importance for the layer characteristics in contrast with the recent analysis of Wunsch. ${ }^{19}$

These ideas are further supported by recent experiments on the dynamics of a vertical columnar vortex pair performed precisely with the aim of shedding light on the layering phenomenon of strongly stratified flows. ${ }^{20}$ These experiments show that the vortex pair, created by closing long vertical flaps, is sliced into layers of pancake dipoles as a result of a new instability, which we have called the zigzag instability. Linear stability analyses ${ }^{21,22}$ of a columnar vortex 
pair have revealed that, for small horizontal Froude number and in the inviscid limit, the growth rate of this zigzag instability is self-similar with respect to $k_{z} U / N$, where $k_{z}$ is the vertical wave number. This self-similarity implies that the most amplified wavelength, controlling the layer thickness, is proportional to the buoyancy length $U / N$ when $F_{h} \ll 1$ and in the nondiffusive limit. As a result, the maximum growth rate is independent of the stratification parameter $N$.

Interestingly, vortex structures with a vertical size scaling like $U / N$ have been also reported in stratified TaylorCouette flow ${ }^{23-25}$ in the strongly stratified regime. Like the previous studies, this experiment is characterized by a vertically uniform basic state.

In an attempt to explain these surprising results, we revisit in this paper the RMWL scaling analysis for the nonlinear dynamics of pancake vortices in strongly stratified fluids. In Sec. II, we first nondimensionalize the Euler equations when the horizontal Froude number $F_{h}=U / N L_{h}$ is small following the RMWL scaling analysis. However, we depart from the RMWL scaling by making no assumption on the magnitude of the aspect ratio $\delta=L_{v} / L_{h}$, where $L_{v}$ is the vertical lengthscale. The straightforward observation that seems not to have been made before is that the equations at leading order in $F_{h}$ are self-similar in $z N / U$, where $z$ is the vertical coordinate, implying that $L_{v}=U / N$. Of course, this self-similarity would be broken if vertical lengthscales were externally imposed by boundary or initial conditions. Some implications of the scaling law of the vertical scale for strongly stratified flows are discussed in Sec. III. In particular, it seems to account for the $k_{z}^{-3}$ power law of the vertical spectrum of horizontal kinetic energy observed in the atmosphere and oceans.

\section{SCALING ANALYSIS}

We use the Boussinesq approximation of an inviscid and nondiffusive stratified fluid, in which density is represented by a constant reference value $\rho_{0}$ except in the buoyancy term. The equations of horizontal and vertical momentum, continuity and density conservation read

$$
\begin{aligned}
& \frac{\partial \boldsymbol{u}_{h}^{\prime}}{\partial t^{\prime}}+\boldsymbol{u}_{h}^{\prime} \cdot \nabla_{h}^{\prime} \boldsymbol{u}_{h}^{\prime}+u_{z}^{\prime} \frac{\partial \boldsymbol{u}_{h}^{\prime}}{\partial z^{\prime}}=-\frac{1}{\rho_{0}} \nabla_{h}^{\prime} p^{\prime}, \\
& \frac{\partial u_{z}^{\prime}}{\partial t^{\prime}}+\boldsymbol{u}_{h}^{\prime} \cdot \nabla_{h}^{\prime} u_{z}^{\prime}+u_{z}^{\prime} \frac{\partial u_{z}^{\prime}}{\partial z^{\prime}}=-\frac{1}{\rho_{0}} \frac{\partial p^{\prime}}{\partial z^{\prime}}-\frac{\rho^{\prime \prime} g}{\rho_{0}}, \\
& \boldsymbol{\nabla}_{h}^{\prime} \cdot \boldsymbol{u}_{h}^{\prime}+\frac{\partial u_{z}^{\prime}}{\partial z^{\prime}}=0, \\
& \frac{\partial \rho^{\prime \prime}}{\partial t^{\prime}}+\boldsymbol{u}_{h}^{\prime} \cdot \nabla_{h}^{\prime} \rho^{\prime \prime}+u_{z}^{\prime} \frac{\partial \rho^{\prime \prime}}{\partial z^{\prime}}+\frac{\partial \bar{\rho}}{\partial z^{\prime}} u_{z}^{\prime}=0,
\end{aligned}
$$

where $t^{\prime}$ is the time, $\left(x^{\prime}, y^{\prime}, z^{\prime}\right)$ Cartesian coordinates with $z^{\prime}$ directed in the vertical, $\boldsymbol{u}_{h}^{\prime}$ the horizontal velocity, $u_{z}^{\prime}$ the vertical velocity, $\nabla_{h}^{\prime}$ the horizontal gradient, $p^{\prime}$ the pressure, $g$ the gravity, $\bar{\rho}\left(z^{\prime}\right)$ a linear ambient density and $\rho^{\prime \prime}\left(\boldsymbol{x}^{\prime}, t^{\prime}\right)$ a perturbation density from this ambient profile. The ambient density gradient defines the Brunt-Väisälä frequency $N$ $=\sqrt{-\left(g / \rho_{0}\right) \partial \bar{\rho} / \partial z^{\prime}}$. Dimensional variables are denoted with primes in anticipation of the existence of the second set of dimensionless variables which will be unprimed.

We define $U$ and $W$ to be the characteristic horizontal and vertical velocity scales, $L_{h}$ and $L_{v}$ to be horizontal and vertical lengthscales and $P$ and $R$ to be the order of magnitude of the pressure and density perturbation. We consider that $U$ and $L_{h}$ are externally imposed while $W, P, R$, and $L_{v}$ need to be determined by scaling arguments. Like Riley et $a l .{ }^{7}$ and Lilly, ${ }^{14}$ we assume herein that $F_{h}=U / N L_{h} \ll 1$, i.e., that the flow is strongly stratified. This fundamental hypothesis differs from the assumption $F_{h}=O(1)$ used in the scaling analysis of Gargett. ${ }^{26}$ Since we are interested in the pancake vortex dynamics, we choose the advective time $T$ $=L_{h} / U$ as characteristic time scale. Riley et al. ${ }^{7}$ have shown that this choice effectively filters out fast internal waves with time scale $1 / N$ from slow horizontal advective motions. The horizontal Froude number may be thus interpreted as the ratio of the characteristic time scale of fast internal waves to that of slow advective horizontal motions. Equivalently, $F_{h}$ can be seen either as the ratio between the buoyancy length $L_{b}=U / N$ and the horizontal lengthscale $L_{h}$, or as a measure of the competition between vertical inertial forces and buoyancy.

The two hypotheses $F_{h} \ll 1$ and $T=L_{h} / U$ lead naturally to the RMWL scaling analysis for pancake vortices. Since our results build upon this analysis, we briefly recall its derivation. Equating the horizontal pressure gradient and inertial terms in the horizontal momentum equation (1) gives the magnitude of the pressure $P=\rho_{0} U^{2}$. The density equation (4) and the continuity equation (3) both impose a constraint on the magnitude of the vertical velocity: $W \leqslant R F_{h} g /\left(\rho_{0} N\right)$ and $W \leqslant U \delta$, respectively, so that $W=\min \left(R F_{h} g /\left(\rho_{0} N\right), U \delta\right)$, where $\delta=L_{v} / L_{h}$ is the aspect ratio. In the following, we shall assume a priori that $L_{v} \geqslant U / N$, then it easily follows that $W=R F_{h} g /\left(\rho_{0} N\right)$. The convenient assumption $L_{v}$ $\geqslant U / N$ will be fulfilled a posteriori and only simplifies the analysis without loss of generality. Inserted in the vertical momentum equation (2), the scaling for the vertical velocity indicates that the vertical inertial terms are $O\left(F_{h}^{2}\right)$ smaller than the buoyancy term. Therefore, since $F_{h} \ll 1$, the only way to balance the vertical pressure gradient is by density perturbations through hydrostatic equilibrium. This gives the order of magnitude of density perturbations

$$
R=\rho_{0} U^{2} /\left(g L_{v}\right)
$$

With these characteristic scales, we define unprimed dimensionless quantities

$\boldsymbol{u}_{h}^{\prime}=U \boldsymbol{u}_{h}, \quad u_{z}^{\prime}=U \frac{F_{h}^{2}}{\delta} u_{z}, \quad \rho^{\prime \prime}=\frac{U^{2} \rho_{0}}{g L_{v}} \rho^{\prime}, \quad p^{\prime}=\rho_{0} U^{2} p$

$x^{\prime}=L_{h} x, \quad y^{\prime}=L_{h} y, \quad z^{\prime}=L_{v} z, \quad t^{\prime}=\frac{L_{h}}{U} t$.

In terms of these new variables, one obtains the RMWL dimensionless system 


$$
\begin{aligned}
& \frac{\partial \boldsymbol{u}_{h}}{\partial t}+\boldsymbol{u}_{h} \cdot \nabla_{h} \boldsymbol{u}_{h}+\frac{F_{h}^{2}}{\delta^{2}} u_{z} \frac{\partial \boldsymbol{u}_{h}}{\partial z}=-\nabla_{h} p, \\
& F_{h}^{2}\left[\frac{\partial u_{z}}{\partial t}+\boldsymbol{u}_{h} \cdot \nabla_{h} u_{z}+\frac{F_{h}^{2}}{\delta^{2}} u_{z} \frac{\partial u_{z}}{\partial z}\right]=-\frac{\partial p}{\partial z}-\rho^{\prime}, \\
& \boldsymbol{\nabla}_{h} \cdot \boldsymbol{u}_{h}+\frac{F_{h}^{2}}{\delta^{2}} \frac{\partial u_{z}}{\partial z}=0, \\
& \frac{\partial \rho^{\prime}}{\partial t}+\boldsymbol{u}_{h} \cdot \nabla_{h} \rho^{\prime}+\frac{F_{h}^{2}}{\delta^{2}} u_{z} \frac{\partial \rho^{\prime}}{\partial z}-u_{z}=0,
\end{aligned}
$$

which is valid for $F_{h} \ll 1$ and $\delta \geqslant F_{h}$. Two nondimensional numbers arise in (7)-(10): The horizontal Froude number and the number $F_{v}=F_{h} \delta^{-1}=U / N L_{v}$, which is equivalent to a vertical Froude number. Note that we have expressed (7)-(10) with $\delta$ and $F_{h}$ instead of using $\delta$ and $F_{v}$ as did Riley et al. ${ }^{7}$ Note also that Lilly ${ }^{14}$ assumes isotropy of the flow, i.e., $\delta=1$, so that only one Froude number appears in his dimensionless equations. Beyond these minor differences, the common and crucial assumption of these scaling analyses is that $F_{v}=F_{h} \delta^{-1} \rightarrow 0$, i.e., $L_{v} \gg U / N$, in the strongly stratified limit. As seen from (7)-(10), purely twodimensional equations for the horizontal velocity with undetermined vertical dependence are then obtained in the strongly stratified limit. In contrast, when spontaneous layering from an initially vertically uniform flow occurs as in the numerical experiments of Herring and Métais ${ }^{9}$ or in the laboratory experiments of Fincham et al., ${ }^{4}$ Billant and Chomaz, ${ }^{20}$ Boubnov et al. and Caton et al., ${ }^{23-25}$ the layer thickness is selected dynamically by the flow itself. Because $L_{v}$ is not imposed by scaling arguments, the a priori assumption $L_{v}$ $\gg U / N$ used by RMWL cannot be made in these cases. Our point of view is rather that the aspect ratio $\delta$ should be considered as a free gauge parameter. In contrast, we emphasize that the scale relation $F_{h} \ll 1$, which is the distinguishing attribute of strongly stratified flows, involves only externally imposed parameters. Thus, without any doubt, the vertical inertial terms in the vertical momentum equation (8) are negligible in the strongly stratified limit $F_{h}=0$ whereas, in the absence of any knowledge of the magnitude of $\delta$ when $F_{h}$ $\rightarrow 0$, we keep at this stage the terms proportional to $F_{h}^{2} / \delta^{2}$ in (7)-(10), even if later they turn out to be negligible

$$
\begin{aligned}
& \frac{\partial \boldsymbol{u}_{h}}{\partial t}+\boldsymbol{u}_{h} \cdot \nabla_{h} \boldsymbol{u}_{h}+\frac{F_{h}^{2}}{\delta^{2}} u_{z} \frac{\partial \boldsymbol{u}_{h}}{\partial z}=-\nabla_{h} p, \\
& 0=-\frac{\partial p}{\partial z}-\rho^{\prime}, \\
& \boldsymbol{\nabla}_{h} \cdot \boldsymbol{u}_{h}+\frac{F_{h}^{2}}{\delta^{2}} \frac{\partial u_{z}}{\partial z}=0, \\
& \frac{\partial \rho^{\prime}}{\partial t}+\boldsymbol{u}_{h} \cdot \nabla_{h} \rho^{\prime}+\frac{F_{h}^{2}}{\delta^{2}} u_{z} \frac{\partial \rho^{\prime}}{\partial z}-u_{z}=0 .
\end{aligned}
$$

The aspect ratio remaining unknown, it could be determined by invoking heuristic arguments such as the dominant bal- ance principle. This principle states that a free gauge should be chosen in order to keep as many terms as possible in the equations and would lead to set $\delta=F_{h}$ in (11)-(14). However, a quite remarkable feature of Eqs. (11)-(14) is that $\delta$ appears only through the vertical Froude number $F_{v}=F_{h} / \delta$ suggesting the existence of self-similarity. In the following, we shall demonstrate that (11)-(14) indeed possess a supplementary group of invariance from which derives rigorously the scaling law $\delta=F_{h}$. To this end, we rewrite the Eqs. (11)-(14) in dimensional form

$$
\begin{aligned}
& \frac{\partial \boldsymbol{u}_{h}^{\prime}}{\partial t^{\prime}}+\boldsymbol{u}_{h}^{\prime} \cdot \nabla_{h}^{\prime} \boldsymbol{u}_{h}^{\prime}+u_{z}^{\prime} \frac{\partial \boldsymbol{u}_{h}^{\prime}}{\partial z^{\prime}}=-\frac{1}{\rho_{0}} \nabla_{h}^{\prime} p^{\prime}, \\
& 0=-\frac{1}{\rho_{0}} \frac{\partial p^{\prime}}{\partial z^{\prime}}-\frac{\rho^{\prime \prime} g}{\rho_{0}}, \\
& \boldsymbol{\nabla}_{h}^{\prime} \cdot \boldsymbol{u}_{h}^{\prime}+\frac{\partial u_{z}^{\prime}}{\partial z^{\prime}}=0, \\
& \frac{\partial \rho^{\prime \prime}}{\partial t^{\prime}}+\boldsymbol{u}_{h}^{\prime} \cdot \nabla_{h}^{\prime} \rho^{\prime \prime}+u_{z}^{\prime} \frac{\partial \rho^{\prime \prime}}{\partial z^{\prime}}-N^{2} \frac{\rho_{0}}{g} u_{z}^{\prime}=0 .
\end{aligned}
$$

One can readily remark that Eqs. (15)-(18) are invariant under the following group of transformations:

$$
N=N^{*} / \alpha, \quad z^{\prime}=\alpha z^{\prime *}, \quad u_{z}^{\prime}=\alpha u_{z}^{\prime *}, \quad \rho^{\prime \prime}=\frac{1}{\alpha} \rho^{\prime \prime *}
$$

where $\alpha$ is a constant and the other variables remain unchanged. This means that the time evolution of a solution of (15)-(18) for a given Brunt-Väisälä frequency $N$, starting from a vertically uniform state (with no uniform vertical velocity) and for which no vertical lengthscales are imposed by boundary conditions, can be deduced from the solution for any other frequency $N^{*}$, for instance $N^{*}=1$, according to

$$
\begin{aligned}
& \boldsymbol{u}_{h}^{\prime}=\boldsymbol{u}_{h}^{\prime *}(x, y, z N, t), \\
& u_{z}^{\prime}=\frac{1}{N} u_{z}^{\prime *}(x, y, z N, t), \\
& \rho^{\prime \prime}=N \rho^{\prime \prime} *(x, y, z N, t) .
\end{aligned}
$$

The group of invariance (19) implies that when $N$ increases, the vertical lengthscale decreases inversely proportionally, i.e., $L_{v} \propto 1 / N$, while the time evolution and the spatial structure in the horizontal plane remains identical. Dimensional considerations impose further that $L_{v}=U / N$, i.e., $\delta=F_{h}$. It is also noteworthy that the vertical velocity amplitude decreases inversely proportionally to $N$ while density perturbations increase linearly with $N$. The self-similarity (20)-(22) explains the observations of Herring and Métais ${ }^{9}$ in which three strongly stratified numerical experiments starting from the same initial condition but with different Brunt-Väisälä frequencies were observed to evolve identically with time. It also accounts for the results of the stratified Taylor-Couette experiments $^{23-25}$ and the linear stability analysis of a colum- 
nar vortex pair. $^{22}$ In the latter study, an increase of $N$ for $F_{h} \ll 1$ indeed leads to a decrease of the most amplified wavelength $\lambda$ of the zigzag instability according to $\lambda \propto U / N$ while the maximum growth rate remains the same.

The self-similarity (20)-(22) not only explains the scaling laws observed by Herring and Métais ${ }^{9}$ and Billant and Chomaz $^{22}$ for linear instabilities but also extends them to the full nonlinear dynamic of any strongly stratified flow for which no vertical lengthscales are imposed by initial or boundary conditions.

It is important to note that the transformation group (19) leaves invariant the equations of motion only if the vertical acceleration term is neglected, so that the self-similarity (20)-(22) is exact only in the limit $F_{h}=0$. The results of Herring and Métais ${ }^{9}$ and Billant and Chomaz $^{22}$ for particular flow configurations indicates, however, that there is a finite domain in $F_{h}$ over which the self-similarity is approximately valid provided that $F_{h} \ll 1$. Furthermore, although it is strictly valid only if no vertical lengthscales are prescribed by initial or boundary conditions, the self-similarity should hold in practice if the imposed vertical lengthscales are much larger than $U / N$.

Using the appropriate vertical scale $L_{v}=U / N$, the dimensionless quantities defined in (6) become

$\boldsymbol{u}_{h}^{\prime}=U \boldsymbol{u}_{h}, \quad u_{z}^{\prime}=U F_{h} u_{z}, \quad \rho^{\prime \prime}=\frac{U N \rho_{0}}{g} \rho^{\prime}, \quad p^{\prime}=\rho_{0} U^{2} p$,

$x^{\prime}=L_{h} x, \quad y^{\prime}=L_{h} y, \quad z^{\prime}=\frac{U}{N} z, \quad t^{\prime}=\frac{L_{h}}{U} t$,

and the corresponding dimensionless equations are obtained by simply setting $\delta=F_{h}$ in the RMWL dimensionless systems (7)-(10)

$$
\begin{aligned}
& \frac{\partial \boldsymbol{u}_{h}}{\partial t}+\boldsymbol{u}_{h} \cdot \nabla_{h} \boldsymbol{u}_{h}+u_{z} \frac{\partial \boldsymbol{u}_{h}}{\partial z}=-\nabla_{h} p, \\
& F_{h}^{2}\left[\frac{\partial u_{z}}{\partial t}+\boldsymbol{u}_{h} \cdot \nabla_{h} u_{z}+u_{z} \frac{\partial u_{z}}{\partial z}\right]=-\frac{\partial p}{\partial z}-\rho^{\prime}, \\
& \boldsymbol{\nabla}_{h} \cdot \boldsymbol{u}_{h}+\frac{\partial u_{z}}{\partial z}=0, \\
& \frac{\partial \rho^{\prime}}{\partial t}+\boldsymbol{u}_{h} \cdot \nabla_{h} \rho^{\prime}+u_{z} \frac{\partial \rho^{\prime}}{\partial z}-u_{z}=0 .
\end{aligned}
$$

We see that, even if the vertical velocity is small $u_{z}^{\prime}$ $=F_{h} U u_{z}$, vertical transport and vertical velocity divergence terms are of order one in (24)-(27) because the smallness of the vertical velocity is exactly balanced by the largeness of vertical gradients $\partial / \partial z^{\prime}=\left(1 / F_{h} L_{h}\right) \partial / \partial z$. Only the vertical acceleration in $(25)$ can be neglected when $F_{h} \ll 1$. Similar equations, for which the hydrostatic balance is valid in first approximation, arise naturally in many geophysical situations when the horizontal lengthscale is much larger than the vertical lengthscale.

The solution of (24)-(27) could be sought as an asymptotic expansion in $F_{h}^{2}$. The asymptotic solution would read in dimensional form

$$
\begin{aligned}
\left(\frac{\boldsymbol{u}_{h}^{\prime}}{U}, \frac{u_{z}^{\prime}}{U F_{h}}, \frac{\rho^{\prime \prime} g}{\rho_{0} N U}\right)= & f_{0}\left(\frac{x^{\prime}}{L_{h}}, \frac{y^{\prime}}{L_{h}}, \frac{z^{\prime} N}{U}, \frac{t^{\prime} U}{L_{h}}\right) \\
& +F_{h}^{2} f_{2}\left(\frac{x^{\prime}}{L_{h}}, \frac{y^{\prime}}{L_{h}}, \frac{z^{\prime} N}{U}, \frac{t^{\prime} U}{L_{h}}\right)+\cdots
\end{aligned}
$$

While the whole solution is not exactly self-similar except if $F_{h}=0$, one can remark that each separate order, $f_{0}, f_{2}$, etc., is self-similar.

\section{DISCUSSION}

Equations (24)-(27) found above describe a new threedimensional dynamic that deserves comparison with twodimensional and three-dimensional dynamics of homogeneous flows. Three-dimensional motions of an inviscid homogeneous flow may be easily understood and described by referring to the Kelvin and Helmholtz theorems stating, respectively, that vorticity fluxes through material surfaces are conserved and that vorticity tubes are, therefore, simply advected. In contrast, in stratified flows, the vorticity is no longer only transported since the baroclinic torque may create vorticity. The only invariants we are left with in the Boussinesq approximation are the mass, the density and the scalar quantity $\Pi=\boldsymbol{\nabla} \times \boldsymbol{u} \cdot \boldsymbol{\nabla} \rho$ known as Ertel's potential vorticity.

When the flow is strongly stratified, the deformations of the isopycnal surfaces are of the order $F_{v} U / N$, therefore if we assume, like RMWL, that $F_{v} \ll 1$, then the isopycnal surfaces are flat at leading order. The Ertel theorem thus implies conservation of the vertical vorticity and mass conservation imposes that the velocity be divergent-free in the horizontal plane. Therefore, the dynamics in each layer is governed by the incompressible two-dimensional Euler equations. This result brought Lilly ${ }^{14,27}$ to argue that stratified turbulence should be similar to two-dimensional turbulence. Although this conjecture could be actually true since some studies of stratified flows have shown enhanced upscale energy transfer in the horizontal like in two-dimensional turbulence, ${ }^{3,7-9}$ we see from the present analysis that the proof of Lilly based on the RMWL scaling is not valid because the intrinsic dynamics of strongly stratified flows should be such that $L_{v}$ $=U / N$ when no vertical lengthscales are externally imposed. This means that the deformations of isopycnal surfaces are comparable to the vertical size of eddies.

The study of strongly stratified turbulence using Eqs. (24)-(27) in the limit $F_{h} \rightarrow 0$ is beyond the scope of the present paper. We simply note that the turbulence of strongly stratified fluids is likely to be different from two-dimensional turbulence because vertical stretching of vertical vorticity is possible and the horizontal velocity is no longer divergencefree. In addition, Ertel's potential vorticity conserved by (24)-(27) does not approximate the vertical vorticity but a complex quantity when $F_{h}=0$ 


$$
\begin{aligned}
\Pi=\boldsymbol{\nabla} \times \boldsymbol{u} \cdot \boldsymbol{\nabla} \rho= & \left(-1+\frac{\partial \rho^{\prime}}{\partial z}\right)\left(\frac{\partial u_{y}}{\partial x}-\frac{\partial u_{x}}{\partial y}\right)-\frac{\partial \rho^{\prime}}{\partial x} \frac{\partial u_{y}}{\partial z} \\
& +\frac{\partial \rho^{\prime}}{\partial y} \frac{\partial u_{x}}{\partial z}+O\left(F_{h}^{2}\right) .
\end{aligned}
$$

The contributions of vertical and horizontal components of vorticity are of the same importance in (29). However, the horizontal vorticity is dominated by vertical shear of horizontal velocity, the horizontal shear of vertical velocity being negligible. It is unknown whether or not the conservation of this quantity implies an inverse cascade of energy towards large scales as in two-dimensional turbulence. ${ }^{28}$ Nevertheless, a number of simple but important implications for the physics of strongly stratified flows can be stated by examination of (24)-(27) in the limit $F_{h}=0$.

\section{A. Slow internal waves and quasi-horizontal vortices}

The first interesting property is that Eqs. (24)-(27) contain both quasi-horizontal motions and slow internal gravity waves even in the limit $F_{h}=0$. If one inserts a wave solution of the form

$$
\left(\boldsymbol{u}_{h}, u_{z}, p, \rho^{\prime}\right) \propto e^{i(\boldsymbol{k} \cdot \boldsymbol{x}-\omega t)}
$$

in (24)-(27) and linearizes in the usual fashion, then the following dimensionless dispersion relation is obtained when $F_{h}=0$ :

$$
\omega^{2}=\frac{k_{h}^{2}}{k_{z}^{2}},
$$

where $\boldsymbol{k}_{h}$ and $k_{z}$ are the horizontal and vertical wave numbers. This dispersion relation is the approximation of the well-known dimensionless dispersion relation of internal gravity waves $\omega^{2}=\boldsymbol{k}_{h}^{2} /\left(\boldsymbol{k}_{h}^{2}+k_{z}^{2}\right)$ when the vertical wave number is much larger than the horizontal one $k_{z} \gg k_{h}$. The existence of such slow internal waves is readily explained by the fact that internal waves have their frequency in the range $[0, N]$. Thus, even if $N \gg U / L_{h}$, there exist slow internal waves which have a frequency comparable to $U / L_{h}$ (the corresponding dimensional vertical wave number is $k_{z}$ $=O(N / U))$. These waves can, therefore, intimately interact with horizontal advective motions.

\section{B. Approximate equipartition of energy}

Another important property is that kinetic and potential energies are of the same order of magnitude for a strongly stratified flow with a vertical lengthscale $L_{v}=U / N$. To see this, we introduce the total energy integrated over the entire three-dimensional domain

$$
E=\iiint(\mathrm{KE}+\mathrm{PE}) d x d y d z,
$$

which is conserved by (1)-(4). $\mathrm{KE}=\frac{1}{2}\left(\boldsymbol{u}_{h}^{2}+u_{z}^{2}\right)$ is the kinetic energy and $\mathrm{PE}=\frac{1}{2}\left(\rho^{\prime} g / \rho_{0} N\right)^{2}$ is the potential energy. The order of magnitude of the kinetic energy is $U^{2}+U^{2} F_{h}^{2} F_{v}^{2}$. Using the scale for density perturbation $R=\rho_{0} U^{2} / g L_{v}$ given by (5), the magnitude of the potential energy PE is $U^{2} F_{v}^{2}$. Kinetic and potential energies are, therefore, of the same order when the vertical lengthscale $L_{v}$ is equal to the buoyancy lengthscale $U / N$. Thus, as for internal gravity waves which achieve an exact equipartition between kinetic and potential energies, there is also an approximate equipartition for quasi-horizontal motions described by (24)-(27) when $F_{h} \rightarrow 0$.

\section{Diffusive effects}

In addition to non-zero horizontal Froude number effects, viscous and diffusive effects may also alter the selfsimilarity. If viscous effects are introduced in (1)-(4), it appears that dissipation is mostly due to vertical shear $\partial^{2} \boldsymbol{u}_{h} / \partial z^{2}$ since the vertical lengthscale $U / N$ is much thinner that the horizontal one $L_{h}$. This feature is in agreement with experimental ${ }^{4}$ and numerical observations. ${ }^{8,9}$ For instance, Fincham et al. ${ }^{4}$ have reported in two particular experiments that the vertical shearing is responsible for more than $90 \%$ of the dissipation budget of the flow behind a rake of vertical flat-plates. In these two experiments, the initial horizontal Froude numbers based on the plate width were below unity: $F_{h}=0.26$ and $F_{h}=0.46$, so that the present scaling analysis should be applicable in first approximation.

Using the scale $L_{v}=U / N$, we can compare viscous diffusion by vertical shear to inertial effects by means of the modified Reynolds number

$$
\mathrm{Re}=\frac{\text { inertial force }}{\text { viscous force }}=\frac{U^{2} / L_{h}}{v U / L_{v}^{2}}=F_{h}^{2} \operatorname{Re}_{h},
$$

where $\operatorname{Re}_{h}=U L_{h} / v$ is the usual Reynolds number based on the horizontal lengthscale, $v$ being the kinematic viscosity. The effective Reynolds number Re is, therefore, reduced by a factor $F_{h}^{2}$ relative to the classical Reynolds number $\operatorname{Re}_{h}$. Viscous effects will become important and break the selfsimilarity when $\operatorname{Re}<1$, i.e., when $F_{h}^{2}<1 / \operatorname{Re}_{h}$. Since the horizontal Froude number must be small, the self-similarity is therefore expected to hold in the range

$$
\frac{1}{\operatorname{Re}_{h}^{1 / 2}} \ll F_{h} \ll 1,
$$

that is to say, only if the horizontal Reynolds number is quite large. A similar argument for density diffusion effects shows that they will become important when $\operatorname{Re}_{h} \mathrm{Sc}<1 / F_{h}^{2}$, where $\mathrm{Sc}=v / D$ is the Schmidt number, $D$ being the molecular diffusivity. In the case of laboratory experiments using salt water as stratifying agent, the Schmidt number is large so that viscous, rather than diffusive, effects will be the limiting factor for the self-similarity to be observed.

The scaling relation (33) indicates that the fraction of dissipation by vertical shear $V$ should be related to the horizontal Froude number by

$$
F_{h} \sim \sqrt{1-V},
$$

for small $F_{h}$. In the case of the two experiments by Fincham et al. ${ }^{4}$ mentioned above, the measured fraction, $V \approx 90 \%$ should, therefore, correspond to a horizontal Froude number of the order of 0.3. This value is in good agreement with the actual Froude number values: $F_{h}=0.26$ and $F_{h}=0.46$, 
knowing that (35) is only an order of magnitude relation. Furthermore, this fraction of dissipation by vertical shear can be seen to increase in time and to rapidly account for the total dissipation. ${ }^{4}$ This feature agrees also qualitatively with (33) since the horizontal Froude number decreases with time as the kinetic energy decays and as the layers grow horizontally. In these two experiments, the corresponding Reynolds numbers based on the plate width are about 386 for $F_{h}$ $=0.26$ and 1545 for $F_{h}=0.46$ so that the constraint (34) is initially approximately fulfilled.

\section{Rotation effects}

Geophysical flows, especially slow waves and largescale flows, are not only influenced by a stable stratification but also by the planetary rotation. When the Coriolis force: $2 \Omega \boldsymbol{e}_{z} \times \boldsymbol{u}_{h}^{\prime}$, where $\Omega$ is the rotation rate with an arbitrary magnitude, is included in the left-hand side of Eq. (1), the scaling analysis of Sec. II remains valid. Most strikingly, the group of transformation (19) still holds in the limit $F_{h}=0$ regardless of the value of the Rossby number Ro $=U / \Omega L_{h}$. This means that, in strongly stratified and rotating flows, the vertical lengthscale should be always inversely proportional to the Brunt-Väisälä frequency, $L_{v} \propto 1 / N$. However, to satisfy dimensionality, we have now an infinite number of possible choices since $U / N$ and $\Omega L_{h} / N$ have both the dimension of a length. The vertical scale can be nonetheless written in the form $L_{v}=U \mathcal{F}(\mathrm{Ro}) / N$, where $\mathcal{F}$ is a nondimensional unknown function of the Rossby number Ro. The present analysis have shown that $\mathcal{F}(\mathrm{Ro}) \rightarrow 1$ as $\mathrm{Ro} \rightarrow \infty$. In the opposite limit, i.e., the quasigeostrophic limit (strongly stratified and rapidly rotating flows), we know from the quasigeostrophic theory ${ }^{29}$ that $L_{v}=\Omega L_{h} / N$ so that $\mathcal{F}(\mathrm{Ro})$ $\rightarrow 1$ /Ro when Ro $\rightarrow 0$. The transition between these two limits is likely to be continuous. It is noteworthy that the vertical lengthscales in both limits are consistent: The vertical lengthscale in the quasigeostrophic case can be indeed simply obtained by setting $U=\Omega L_{h}$ in the scaling law $L_{v}$ $=U / N$ of the nonrotating strongly stratified case.

\section{E. Vertical spectra of horizontal kinetic energy and density perturbations}

We finish by deriving an important and simple consequence of the present similarity analysis for the vertical power spectrum of horizontal kinetic energy and density perturbations. Although the scaling analysis is exact for flows with no vertical length scales imposed by boundary or initial conditions, we may conjecture that for turbulent flows the scaling holds for all the length scales within an "inertial", range, i.e., from the injection lengthscale to the dissipation lengthscale. Such a strong hypothesis is classically made in cascade theories of turbulence. In this way, the generated vortices are assumed pancake-like with their vertical wave number $k_{z}$ related to their typical horizontal velocity $U$ by $k_{z} \propto N / U$ at any intermediate horizontal lengthscale, the injection lengthscale being "forgotten." A supporting argument is that decoupling instabilities such as the zigzag instability of the vortex pair $^{21,22}$ operate on the eddy turn-over time scale. Therefore, vortices adapt their thickness to their horizontal velocity through decoupling instabilities as fast as nonlinear processes of the cascade. The vertical spectrum of horizontal kinetic energy per unit mass is defined by $\frac{1}{2} U^{2}$ $=\int_{0}^{\infty} E\left(k_{z}\right) d k_{z}$ so that $E\left(k_{z}\right) \propto U^{2} / \Delta k_{z}$. Since we consider compact eddies and not wavelike disturbances, a classical result of turbulence theory ${ }^{30}$ is that the bandwidth is proportional to the wave number: $\Delta k_{z} \propto k_{z}$. Upon using the above relations, we conclude that $E\left(k_{z}\right)$ should be of the form

$$
E\left(k_{z}\right) \propto N^{2} k_{z}^{-3} .
$$

Likewise, using the scaling (23), we deduce that the vertical spectrum of density perturbations should be of the form $E_{\rho}\left(k_{z}\right) \propto\left(\rho_{0} / g\right)^{2} N^{4} k_{z}^{-3}$. Most interestingly, there are a considerable number of observations of such vertical spectra in the atmosphere ${ }^{31-36}$ and in the oceans. ${ }^{37}$

Various theories, mostly considering internal gravity waves, have been put forward to explain these universal vertical spectra. ${ }^{32,33,38,39}$ They are based on saturation of internal waves, ${ }^{32,33}$ diffusive modeling of nonlinear interaction between gravity waves ${ }^{38}$ or stochastic modeling of the Doppler spreading of vertical wave number by the random winds of the waves. ${ }^{39}$ The theory of saturation of internal waves ${ }^{32,33}$ is the simplest. Interestingly, it also yields the relation $U$ $\propto N / k_{z}$ by arguing that the velocity and density fluctuations are due to internal gravity waves saturated by shear or convective instabilities. This saturation process occurs at large vertical wave number when the wave horizontal velocity $U$ is such that $U \approx \omega / k_{h}=N / k_{z}$, where $\omega$ is the wave frequency. However, two objections can be raised against these hypotheses. First, the linear dispersion relation of internal waves is not expected to be valid when the wave is saturated since nonlinear terms are then of the same order than linear terms. Second, although the dispersion relation is valid for a monochromatic wave, it is extended to a superposition of internal waves.

In addition, one can remark that pancake turbulence might be difficult to distinguish from internal waves. Indeed, the typical frequency of pancake vortices $\omega$ obeys a scaling law $\omega \approx U k_{h} \approx N k_{h} / k_{z}$ resembling the linear dispersion relation of internal waves for large vertical wave number. Furthermore, the group velocity of gravity waves with vertical wave number $k_{z}=O(N / U)$ is $u_{g h}=O(U)$ and $u_{g z}$ $=O\left(F_{h} U\right)$ in the horizontal and vertical direction, respectively, like pancake vortices. Therefore, at first sight, pancake turbulence may resemble internal waves. In any case, the property that $\omega, \boldsymbol{k}_{h}$, and $k_{z}$ are related through the relation dispersion of internal waves as assumed in the empirical Garrett-Munk spectra, ${ }^{40}$ is not sufficient to prove that the motions under consideration are internal waves.

\section{CONCLUSIONS}

In this paper, we have derived a new scaling analysis for vortices in strongly stratified flows built upon the RMWL scaling analysis. Following the latter analysis, the two hypotheses of the present scaling analysis are: The advective time scale is chosen as characteristic time scale and the horizontal Froude number is assumed to be small, $F_{h}=U / N L_{h}$ $\ll 1$, where $U$ and $L_{h}$ are imposed externally. However, con- 
trary to the RMWL analysis, we have considered the vertical lengthscale $L_{v}$ as a free lengthscale not externally imposed. Accordingly, no a priori assumption on the magnitude of the vertical lengthscale $L_{v}$ has been made. In the strongly stratified limit, $F_{h}=0$, the inviscid Boussinesq equations have been shown to become self-similar with respect to the variable $z N / U$ when no vertical lengthscales are externally imposed through boundary or initial conditions. These conditions pertain to the numerical experiments of Herring and Métais, ${ }^{9}$ laboratory experiments of Fincham et al. ${ }^{4}$ Billant and Chomaz, ${ }^{20}$ Boubnov et al. and Caton et al. ${ }^{23-25}$ This self-similarity arises because the acceleration term in the vertical momentum equation is negligible when $F_{h} \ll 1$. It implies that the vertical scale of the motion is $L_{v}=U / N$ making the scaling determinate without any further assumption. This scaling law explains recent experimental and numerical results ${ }^{9,21-25}$ and can simply account for the $N^{2} k_{z}^{-3}$ power law of the vertical spectrum of horizontal kinetic energy observed in the atmosphere and oceans.

Using the appropriate vertical lengthscale, a new nondimensional set of equations for strongly stratified flows has been obtained. It is particularly noteworthy that these equations describe a mixture of pancake vortices and slow internal waves whose dynamics cannot be separated. Furthermore, these equations do not tend to two-dimensional equations in the limit of strong stratification indicating that strongly stratified turbulence should be different from twodimensional turbulence. However, much remains to be done to fully understand the dynamics described by these equations.

\section{ACKNOWLEDGMENTS}

This work has benefited from stimulating discussions with Claude Sidi. We wish to thank Laurette Tuckerman for her fruitful comments and careful reading of the manuscript.

${ }^{1}$ J. T. Lin and Y. H. Pao, "Wakes in stratified fluids: A review," Annu. Rev. Fluid Mech. 11, 317 (1979).

${ }^{2}$ E. J. Hopfinger, "Turbulence in stratified fluids: A review," J. Geophys. Res. 92, 5287 (1987).

${ }^{3}$ J. M. Chomaz, P. Bonneton, A. Butet, and E. J. Hopfinger, "Vertical diffusion in the far wake of a sphere moving in a stratified fluid," Phys. Fluids A 5, 2799 (1993).

${ }^{4}$ A. M. Fincham, T. Maxworthy, and G. R. Spedding, "Energy dissipation and vortex structure in freely decaying stratified grid turbulence,' Dyn. Atmos. Oceans 23, 171 (1996).

${ }^{5}$ G. R. Spedding, F. K. Browand, and A. M. Fincham, “Turbulence, similarity scaling and vortex geometry in the wake of a sphere in a stablystratified fluid,' J. Fluid Mech. 314, 53 (1996).

${ }^{6}$ G. R. Spedding, "The evolution of initially turbulent bluff-body wakes at high internal Froude number,"' J. Fluid Mech. 337, 283 (1997).

${ }^{7}$ J. J. Riley, R. W. Metcalfe, and M. A. Weissman, "Direct numerical simulations of homogeneous turbulence in density stratified fluids," in Nonlinear Properties of Internal Waves, edited by B. J. West, AIP Conf. Proc. 76, 79 (1981).

${ }^{8}$ O. Métais and J. H. Herring, "Numerical simulations of freely evolving turbulence in stably stratified fluids," J. Fluid Mech. 202, 117 (1989).

${ }^{9}$ J. R. Herring and O. Métais, "Numerical simulations in forced stably stratified turbulence," J. Fluid Mech. 202, 97 (1989).

${ }^{10}$ Y. Kimura and J. R. Herring, "Diffusion in stably stratified turbulence," J. Fluid Mech. 328, 253 (1996).

${ }^{11}$ M. C. Gregg, "Diapycnal mixing in the thermocline: A review," J. Geophys. Res. 92, 5249 (1987).
${ }^{12}$ J. R. Alisse and C. Sidi, "Experimental probability density functions of small-scale fluctuations in the stably stratified atmosphere,' J. Fluid Mech. 402, 137 (2000).

${ }^{13}$ J. J. Riley and M.-P. Lelong, "Fluid motions in the presence of strong stable stratification," Annu. Rev. Fluid Mech. 32, 613 (2000).

${ }^{14} \mathrm{D}$. K. Lilly, "Stratified turbulence and the mesoscale variability of the atmosphere,'” J. Atmos. Sci. 40, 749 (1983).

${ }^{15}$ D. C. Stillinger, K. N. Helland, and C. W. Van Atta, "Experiments on the transition of homogeneous turbulence to internal waves in a stratified fluid,' J. Fluid Mech. 131, 91 (1983).

${ }^{16}$ E. C. Itsweire, K. N. Helland, and C. W. Van Atta, "The evolution of grid-generated turbulence in a stably stratified fluid,' J. Fluid Mech. 162, 299 (1986).

${ }^{17}$ J. H. Lienhard and C. W. Van Atta, "The decay of turbulence in thermally stratified flow," J. Fluid Mech. 210, 57 (1990).

${ }^{18}$ F. K. Browand, D. Guyomar, and S.-C. Yoon, "The behavior of a turbulent front in a stratified fluid: Experiments with an oscillating grid," J. Geophys. Res. 92, 5329 (1987).

${ }^{19} \mathrm{~S}$. Wunsch, "Scaling laws for layer formation in stably stratified turbulent flows," Phys. Fluids 12, 672 (2000).

${ }^{20} \mathrm{P}$. Billant and J.-M. Chomaz, "Experimental evidence for a new instability of a vertical columnar vortex pair in a strongly stratified fluid,' J. Fluid Mech. 418, 167 (2000).

${ }^{21}$ P. Billant and J.-M. Chomaz, "Theoretical analysis of the zigzag instability of a vertical columnar vortex pair in a strongly stratified fluid,' J. Fluid Mech. 419, 29 (2000).

${ }^{22} \mathrm{P}$. Billant and J.-M. Chomaz, "Three-dimensional stability analysis of a vertical columnar vortex pair in a stratified fluid,' J. Fluid Mech. 419, 65 (2000)

${ }^{23}$ B. M. Boubnov, E. B. Gledzer, and E. J. Hopfinger, "Stratified circular Couette flow: Instability and flow regimes," J. Fluid Mech. 292, 333 (1995).

${ }^{24}$ B. M. Boubnov, E. B. Gledzer, E. J. Hopfinger, and P. Orlandi, "Layer formation and transitions in stratified circular Couette flow," Dyn. Atmos. Oceans 23, 139 (1996).

${ }^{25}$ F. Caton, B. Janiaud, and E. J. Hopfinger, "Stability and bifurcations in stratified Taylor-Couette flow," J. Fluid Mech. 419, 93 (2000).

${ }^{26} \mathrm{~A}$. E. Gargett, "The scaling of turbulence in the presence of stable stratification,"' J. Geophys. Res. 93, 5021 (1988).

${ }^{27}$ D. K. Lilly, G. Bassett, K. Droegemeir, and P. Bartello, "Stratified turbulence in the atmospheric mesoscales," Theor. Comput. Fluid Dyn. 11, 139 (1998).

${ }^{28}$ R. H. Kraichnan, “Inertial ranges in two-dimensional turbulence," Phys. Fluids 10, 1417 (1967).

${ }^{29}$ J. G. Charney, "Geostrophic turbulence,"' J. Atmos. Sci. 28, 1087 (1971).

${ }^{30} \mathrm{H}$. Tennekes and J. L. Lumley, A First Course in Turbulence (MIT Press, Cambridge, MA, 1972).

${ }^{31}$ R. M. Endlich, R. C. Singleton, and J. W. Kaufman, "Spectral analysis of detailed vertical wind speed profiles," J. Atmos. Sci. 26, 1030 (1969).

${ }^{32}$ E. M. Dewan and R. E. Good, "Saturation and the 'universal' spectrum for vertical profiles of horizontal scalar winds in the atmosphere,' J. Geophys. Res. 91, 2742 (1986).

${ }^{33}$ S. A. Smith, D. C. Fritts, and T. E. VanZandt, "Evidence for a saturated spectrum of atmospheric gravity waves," J. Atmos. Sci. 44, 1404 (1987).

${ }^{34}$ D. C. Fritts, T. Tsuda, T. Sato, S. Fukuao, and S. Kato, "Observational evidence of a saturated gravity waves spectrum in the troposphere and lower stratosphere," J. Atmos. Sci. 45, 1741 (1988).

${ }^{35}$ D. C. Senft and C. S. Gardner, "Seasonal variability of gravity waves activity and spectra in the mesopause region at Urbana," J. Geophys. Res. 96, 17229 (1991).

${ }^{36}$ Y. F. Wu and H. U. Widdel, "Further study of a saturated gravity wave spectrum in the mesosphere," J. Geophys. Res. 96, 9263 (1991).

${ }^{37}$ A. E. Gargett, P. J. Hendricks, T. B. Sanford, T. R. Osborn, and A. J. Williams, "A composite spectrum of vertical shear in the upper ocean," J. Phys. Oceanogr. 11, 1258 (1981).

${ }^{38}$ J. Weinstock, "Saturated and unsaturated spectra of gravity waves and scale-dependent diffusion," J. Atmos. Sci. 47, 2211 (1990).

${ }^{39} \mathrm{C}$. O. Hines, "The saturation of gravity waves in the middle atmosphere. Part II: Development of Doppler-spread theory,'” J. Atmos. Sci. 48, 1360 (1991).

${ }^{40}$ C. Garrett and W. Munk, "Internal waves in the ocean," Annu. Rev. Fluid Mech. 11, 339 (1979) 